\title{
Soft 2D nanoarchitectonics
}

\author{
Katsuhiko Ariga ${ }^{1,2}$, Shun Watanabe ${ }^{2,3}$, Taizo Mori ${ }^{1}$ and Jun Takeya $\mathbb{D}^{1,2}$
}

\begin{abstract}
Nanoarchitectonics is a new paradigm to combine and unify nanotechnology with other sciences and technologies, such as supramolecular chemistry, self-assembly, self-organization, materials technology for manipulation of the size of material objects, and even biotechnology for hybridization with bio-components. The nanoarchitectonic concept leads to the synergistic combination of various methodologies in materials production, including atomic/molecularlevel control, self-organization, and field-controlled organization. The focus of this review is on soft 2D

nanoarchitectonics. Scientific views on soft 2D nanomaterials are not fully established compared with those on rigid 2D materials. Here, we collect recent examples of 2D nanoarchitectonic constructions of functional materials and systems with soft components. These examples are selected according to the following three categories on the basis of 2D spatial density and motional freedom: (i) well-packed and oriented organic 2D materials with rational design of component molecules and device applications, (ii) well-defined assemblies with 2D porous structures as 2D network materials, and (iii) 2D control of molecular machines and receptors on the basis of certain motional freedom confined in two dimensions.
\end{abstract}

\section{Introduction}

Although various scientific disciplines have contributed to developments in functional materials, there is a common consensus concerning methodologies to create functional materials, such as (i) the design, synthesis, and functionalization of unit molecules and materials ${ }^{1-7}$ and (ii) the rational organization of synthesized units into functional architectures ${ }^{8-15}$. Regulation of nanoscale structures and their organization is crucial to synthesize functional materials. As a powerful concept for the science and technology of nanoscale objects, nanotechnology has been accepted as a novel methodology to regulate nanoscale objects. In fact, the observation and characterization of atoms, molecules, and the other nanoscale objects is realized with various techniques in nanotechnology. For example, the manipulation of nanoscale

\footnotetext{
Correspondence: Katsuhiko Ariga (ARIGA.Katsuhiko@nims.go.jp)

${ }^{1}$ World Premier International (WPI) Research Center for Materials

Nanoarchitectonics (MANA), National Institute for Materials Science (NIMS), 1-1

Namiki, Tsukuba 305-0044, Japan

${ }^{2}$ Department of Advanced Materials Science, Graduate School of Frontier

Sciences, The University of Tokyo, 5-1-5 Kahiwanoha, Kashiwa, Chiba 277-8561, Japan
}

Full list of author information is available at the end of the article objects, including molecular machines ${ }^{16}$ and nanocars ${ }^{17}$, can be performed using ultra-sharp tip motions. Great successes and developments in fundamental and applied sciences on the nanoscale have been realized with nanotechnology ${ }^{18-20}$.

However, nanotechnology is not always practical in the construction of useful-sized functional materials from nanoscale units. In this instance, other research disciplines are applied, such as supramolecular chemistry for self-assembly and self-organization, materials science for manipulation of the size of materials, and even biotechnology for hybridization with bio-components. Therefore, it is a useful to create a new paradigm to combine and unify nanotechnology with these other sciences and technologies. This new concept is referred as to nanoarchitectonics (Fig. 1). The nanoarchitectonics concept was initially proposed as a technical term in a conference title: "1st International Symposium on Nanoarchitectonics Using Suprainteractions" in Tsukuba, Japan, in 2000 by Masakazu Aono ${ }^{21,22}$ and is now accepted by various studies from basic sciences to applied environmental and biomedical fields ${ }^{23-29}$. The nanoarchitectonics concept leads to the synergistic 


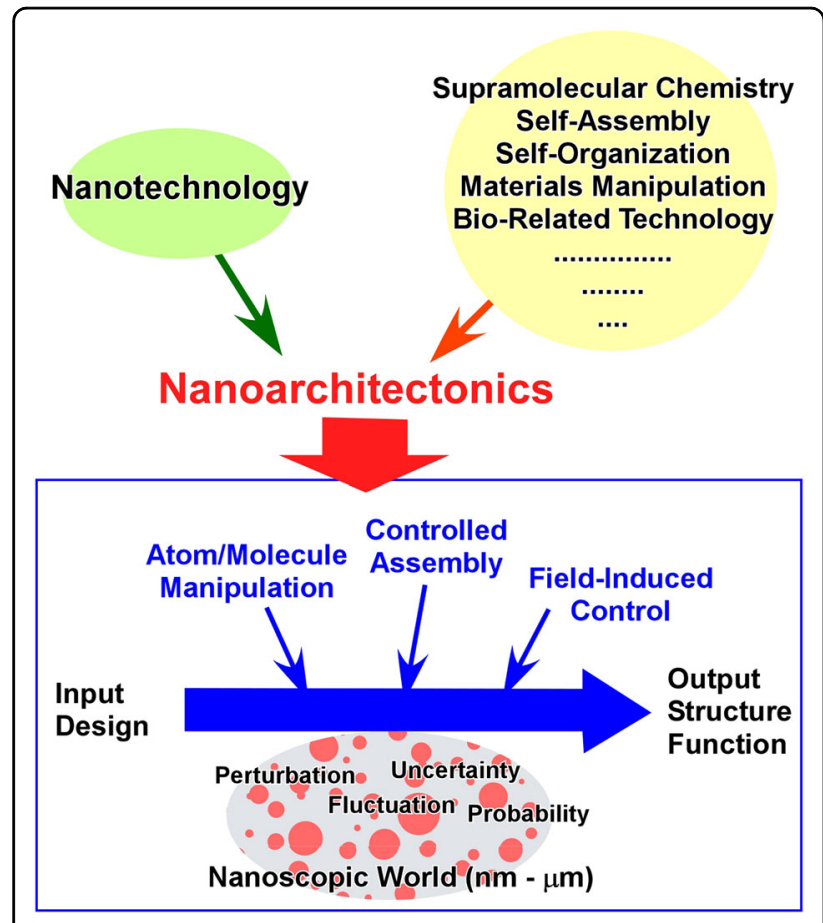

Fig. 1 Outline of the nanoarchitectonic concept

combination of various methodologies in materials production, including atomic/molecular-level control, selforganization, and field-controlled organization ${ }^{30,31}$.

However, nanoscale material production and structure fabrication are fundamentally different from those on the microscale and macroscale. On the latter scales, the strength of interactions (size of interaction envelope) between construction components is large enough to avoid influence from the surrounding environment ${ }^{32}$. Therefore, structures can be precisely obtained according to the intended design in microscopic and macroscopic architectonics. In contrast, this precise design-construction strategy is not always applicable to objects on the nanoscale, because the strength of their interactions is not usually large enough to effectively avoid external disturbances. Various uncontrollable factors, such as thermal fluctuations, static distributions, and certain quantum effects, may have nonnegligible influences on the architecting processes of nanoscale components under ambient conditions. In the latter cases, the harmonization of various factors may be a more appropriate strategy than precise oneby-one construction. Nanoarchitectonic strategies must allow for a level of uncertainty and involve harmonized strategies for component organization. In particular, these features are much more influential when soft matter, such as organic molecules and biological matter, are used as components for materials nanoarchitectonics.
In this short review, we collected recent examples of nanoarchitectonic constructions of functional materials and systems with soft components. The examples were selected from the view of two-dimensional (2D) structural features for two main reasons. The construction of materials confined in 2D nanospaces is much simpler than those in three-dimensional (3D) spaces and may be a much easier target for the starting point of nanoarchitectonics. This is one reason for the selection of $2 \mathrm{D}$ systems. Another reason is originated in the unexplored nature of soft materials in 2D systems that is not achievable in 3D systems. Although 2D materials with rigid components, such as the graphene family, transition metal dichalcogenides, and nanosheets, have attracted much attention ${ }^{33,34}$, scientific views on soft 2D nanomaterials $^{35,36}$ are not fully established. Because of this, here, we briefly introduce, explain, and discuss soft 2D nanoarchitectonics. Because every example cannot be included in this short review, we selected recent examples according to the following three categories on the basis of 2D spatial density and motional freedom. Within 2D confined spaces, component motions are highly restricted, and thus, material packing and their motional behavior seriously affect system design and functional development. The presented examples are classified into (i) wellpacked and oriented states, (ii) mutually linked networks with certain motional flexibilities, and (iii) molecular systems with sufficient motional freedoms. As a more concrete definition, the three categories include (i) wellpacked and oriented organic $2 \mathrm{D}$ materials with rational design of component molecules and device applications, (ii) well-defined assemblies with $2 \mathrm{D}$ porous structures as 2D network materials, and (iii) 2D control of molecular machines and receptors on the basis of considerable motional freedom confined in two dimensions. We hope that exemplifying these three different topics within a unified scientific term, soft 2D nanoarchitectonics, will impress onto readers the great potential of the concept of nanoarchitectonics.

\section{Organic 2D materials and devices}

The assembly of objects into highly symmetric and topological shapes has fascinated the human mind since the beginning of science. The emergence of chemistry along with rapid developments in nanotechnology has made it possible to extend the concept to the nanoworld. Their application in architectural design is of importance particularly for molecules, because the rational construction of a wider variation of nanomaterials can be achieved by directly assembling themselves into desirable sizes, shapes, and functions. At the basic level of selfassembly processes in molecules are molecular building blocks, which are composed of rather simple subunits that are normally synthesized by simple, stepwise covalent- 


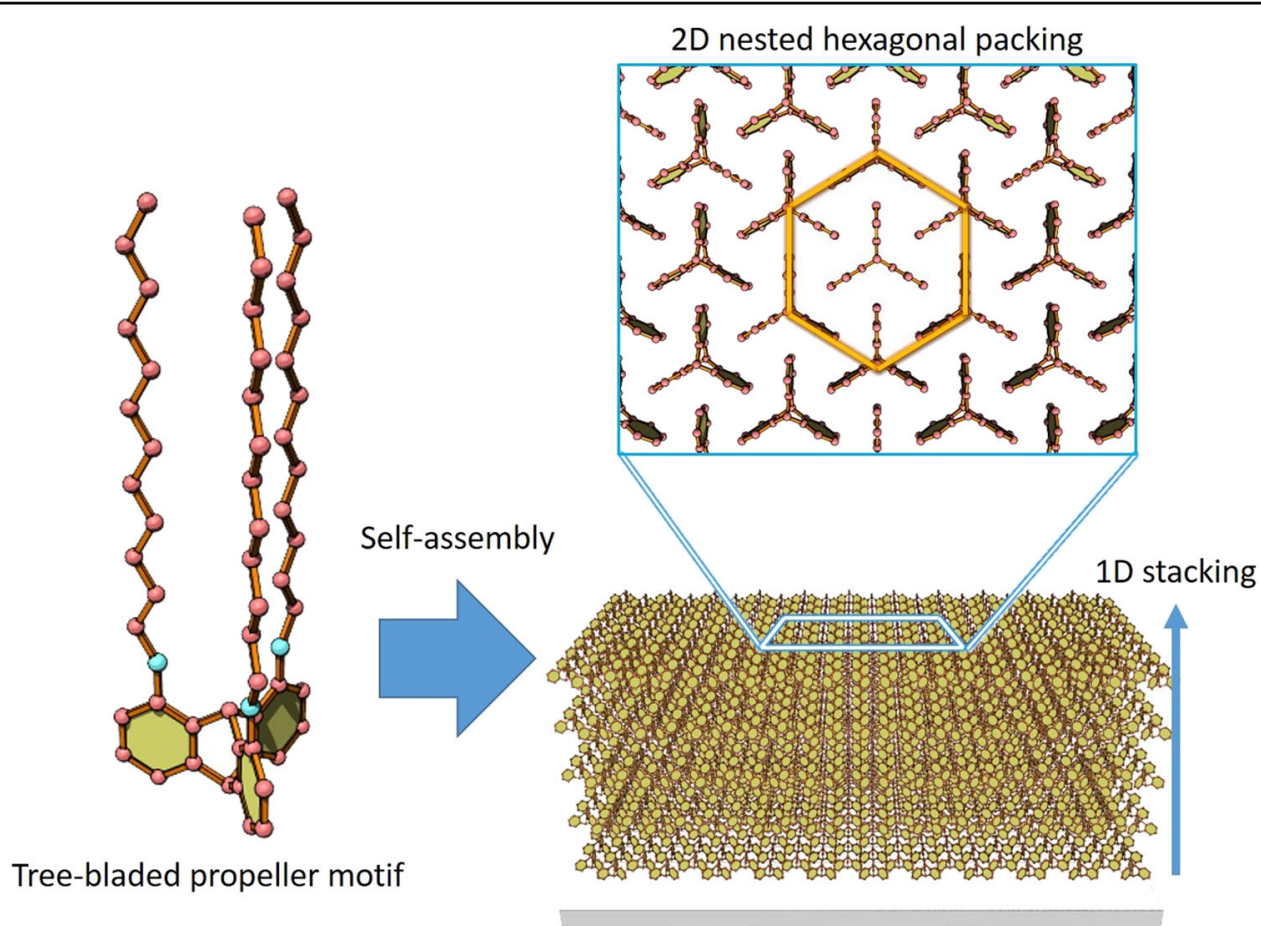

Thin film with completely oriented " $2 D+1 D^{\prime}$ structure

Fig. 2 Domain-boundary-free organic 2D films with triptycene derivatives as nanoarchitecturing units and their 2D $+1 D$ structure

bond chemistry ${ }^{37,38}$. Therefore, not only their own functionality but also the interaction that drives them into a higher order structure is particularly important. Most interactions embedded within molecules are weak, such as hydrogen bonding, $\pi-\pi$ stacking, hydrophilic/hydrophobic interactions, and van der Waals forces ${ }^{39}$. This allows for the controllable production of highly hierarchal nanostructures through low-energy processes, which is particularly important for molecule-based electronics applications.

Very early in the twenty-first century, the science community encountered 2D materials. Recent developments in the synthesis and deposition of 2D nanomaterials have led to the achievement of ideal building blocks for electronic, optoelectronic, and spintronic applications ${ }^{40}$. Graphene and transition metal dichalcogenides have emerged as strong contenders in $2 \mathrm{D}$ materials. Unlike nanosheets, which are composed of a single atomic layer, molecular-based semiconductors have been found to form quasi-2D single crystals ${ }^{41}$. Because molecules are weakly bound by van der Waals interactions, instead of covalent bonds, assembling nanoscale molecules into highly periodic hierarchical structures can be controlled through near-roomtemperature processes. In contrast, the non-covalency limits overlap of the electron wavefunctions, and therefore, charge transport in molecular semiconductors is believed to occur through hopping transport between localized states.

A feature of molecular electronics is the use of molecules to fabricate electronic components. Because a single molecule by itself can have rich electronic or optoelectronic functionalities, the field is widely spread among physics, chemistry, materials science, and engineering. Although a main focus of molecular electronics is molecular assembly, the understanding of how macroscopic functions evolve from the macroscopic to the molecular level remains unclear. To clarify this, domain-boundaryfree organic films with good orientation would be ideal 2D materials for device applications.

Recently, Shoji, Fukushima, and co-workers ${ }^{42}$ proposed a nanoarchitectonic approach with the well-considered design of unit molecules. As illustrated in Fig. 2, they adopted triptycene derivatives as nanoarchitecturing units to realize seamless $2 \mathrm{D}$ structures. The triptycene units have a three-wing propeller motif, and their wings can be integrated into honeycomb structures. The resulting hexagonal space-filling architectonic concept effectively suppressed conformational freedoms, such as translational and rotational motions, to prevent the formation of undesired domain boundaries. Domain-boundary-free organic 2D films can be simply prepared by slow cooling of melted triptycene compounds on a sapphire substrate. The obtained films have a uniform, single-crystal- 
like structure. The formation of a 2D molecular layer can be governed by the dynamic nature, where nucleation, growth, and fusion occur simultaneously. Integration of the wings of the triptycene propeller is likely to propagate

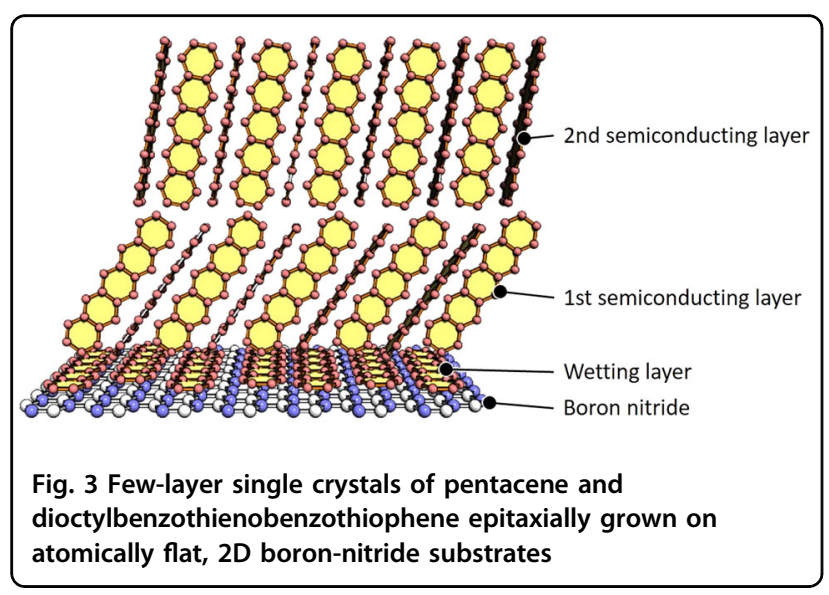

within the 2D plain for an extremely long distance. Similar high-quality 2D structures can be fabricated by spin-coating and vacuum vapor deposition processes, as well as cooling from the isotropic liquid of the triptycene, regardless of the underlying solid substrate. The creation of molecular-level ordered structures on the centimeter level would be very useful for developments in organic dielectric devices and transistors.

We now turn our discussion to molecular electronics and focus particularly on one of the best model studies, that is, the importance of molecular assembly from the viewpoint of condensed matter physics, where the electronic functionality is explained not by extending the properties of a single molecule but by the periodicity of the molecules ${ }^{43}$. Controlling the nanostructures in molecular solids is key to integrating molecular nanomaterials into high-performance devices and applications. Indeed, relatively high field-effect mobilities are achieved in single-crystalline organic semiconductors, which can be

\section{(A) Ink-jet printing}

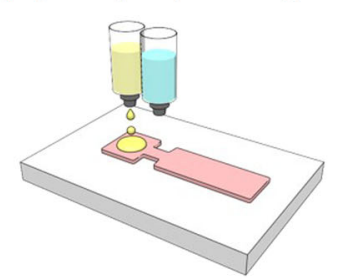

Step. 1

Antisolvent ink is inkjet-printed

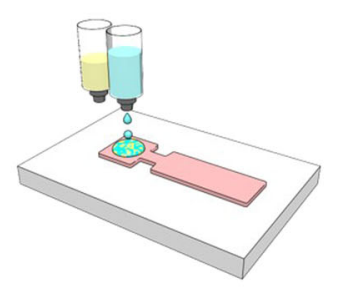

Step. 2

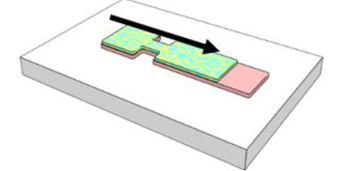

Step. $3 \quad$ Step. 4

Solvent fully evaporates

\section{(B) Fluid-flow}

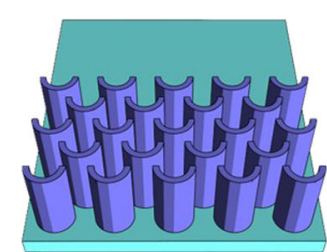

Micropillar-patterned blade

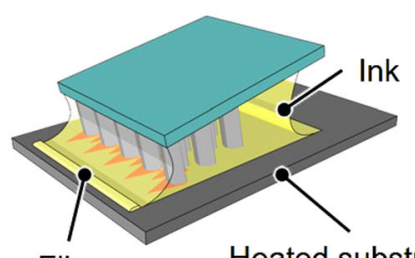

Film
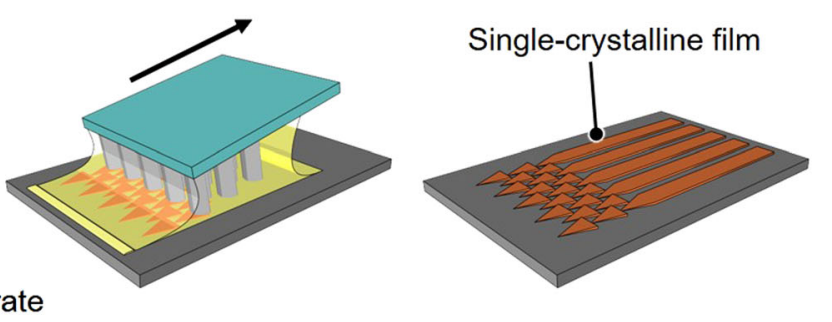

\section{(C) Continuous edge-casting}
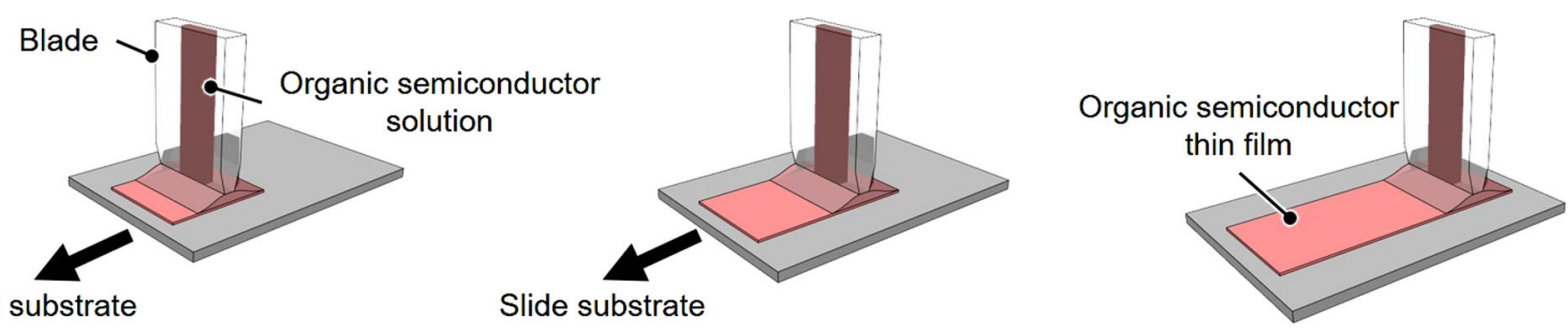

Slide substrate

Slide substrate

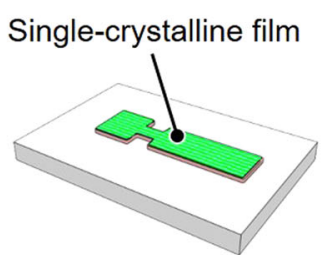

Fig. 4 Meniscus-driven solution processes for a high-quality single-crystalline film. a Ink-jet printing, b fluid-flow, and $\mathbf{c}$ continuous edgecasting 
(A) Supramolecular strand

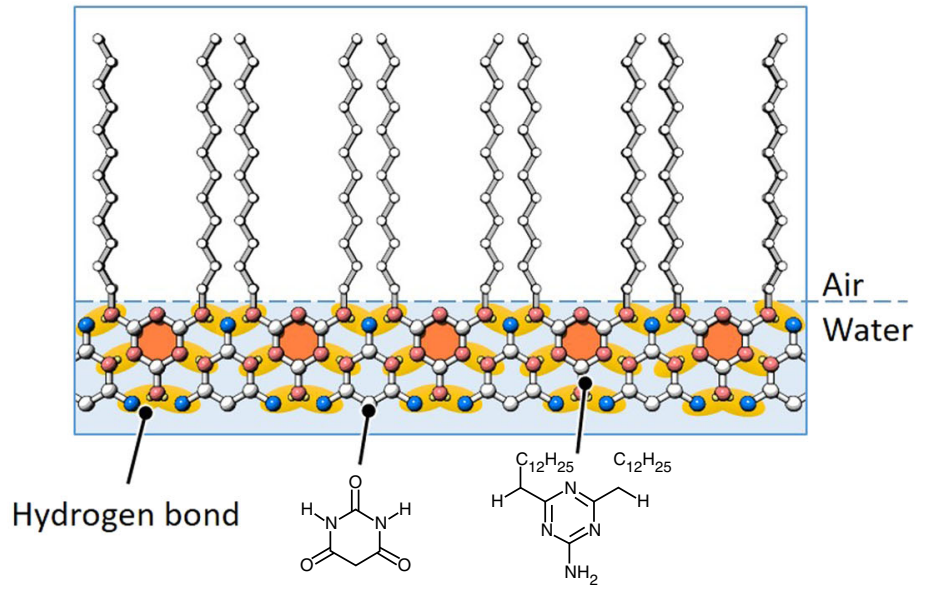

Non-specific interaction

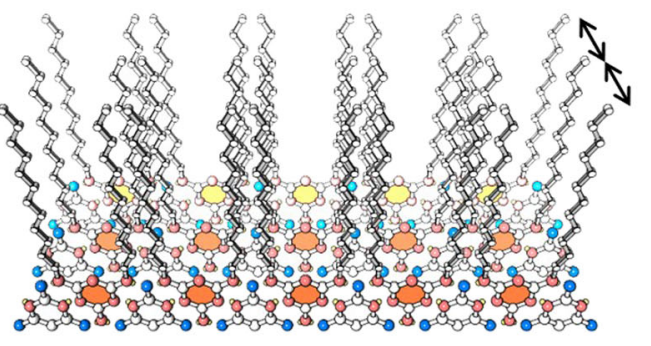

(C) Cross-linked network

(B) Ladder network

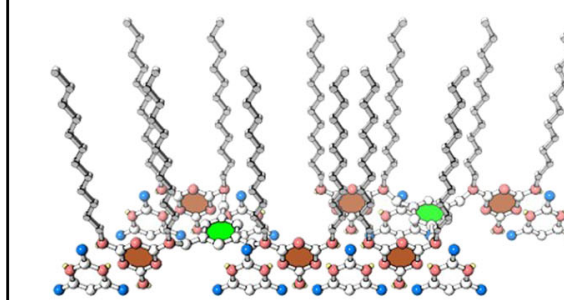

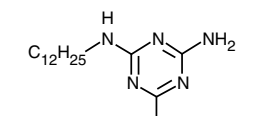

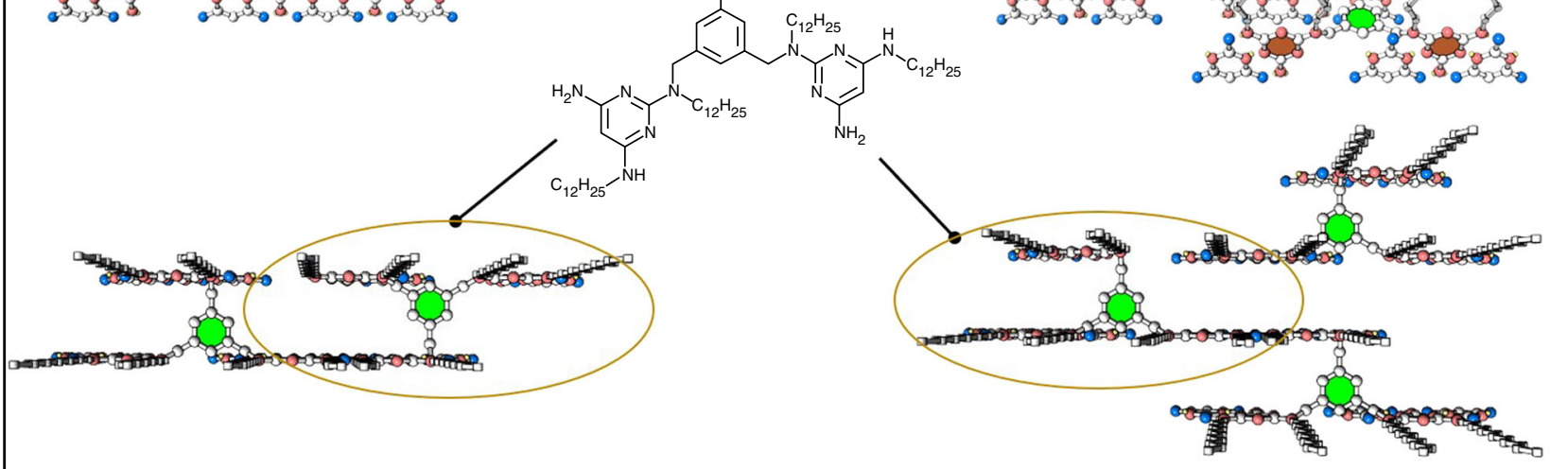

Fig. 5 Supramolecular polymer chains of dialkylmelamine amphiphiles with aqueous barbituric acid at the air-water interface through complementary hydrogen bonding. a Supramolecular strand and $\mathbf{b}$ ladder network c cross-linked network

realized by both designing functional molecules and optimizing crystal growth. We highlight recent developments in state-of-the-art synthetic chemistry and device engineering, where the nanoscale molecular ordering/ packing plays a predominant role in device performance. Many researchers have attempted to achieve wellcontrolled organic single-crystalline semiconductors with reasonably large areal coverage. For instance, organic field-effect transistors using few-layered organic singlecrystalline semiconductors have been reported for several materials. Relatively high mobility is demonstrated in fewlayered single crystals of pentacene and dioctylbenzothienobenzothiophene epitaxially grown on atomically flat boron-nitride substrates (Fig. 3$)^{44}$. However, because such ultrathin crystals can be fabricated only on substrates with a matching lattice constant, their application is considerably limited. Alternatively, fully solution-based processes have been demonstrated to form quasi-2D molecular sheets. Among them, meniscus-driven solution processes, particularly those utilizing a liquid-liquid interface, are believed to be ideal methods to achieve high-quality single-crystalline films (Fig. 4) ${ }^{45-48}$. Importantly, charge carriers in such highly ordered singlecrystalline organic semiconductors undergo truly coherent, band-like charge transport, such as in inorganic materials, as confirmed by observing a negative temperature coefficient for the field-effect mobility and a clear Hall effect, which results in reasonably high mobilities of 


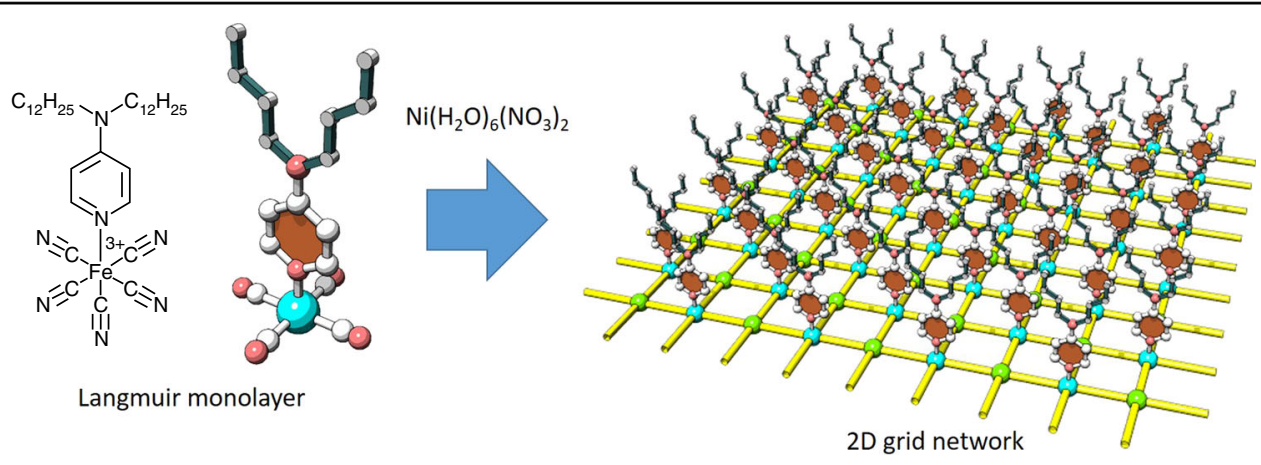

Fig. 6 Two-dimensional coordinate covalent square grid network at the air-water interface

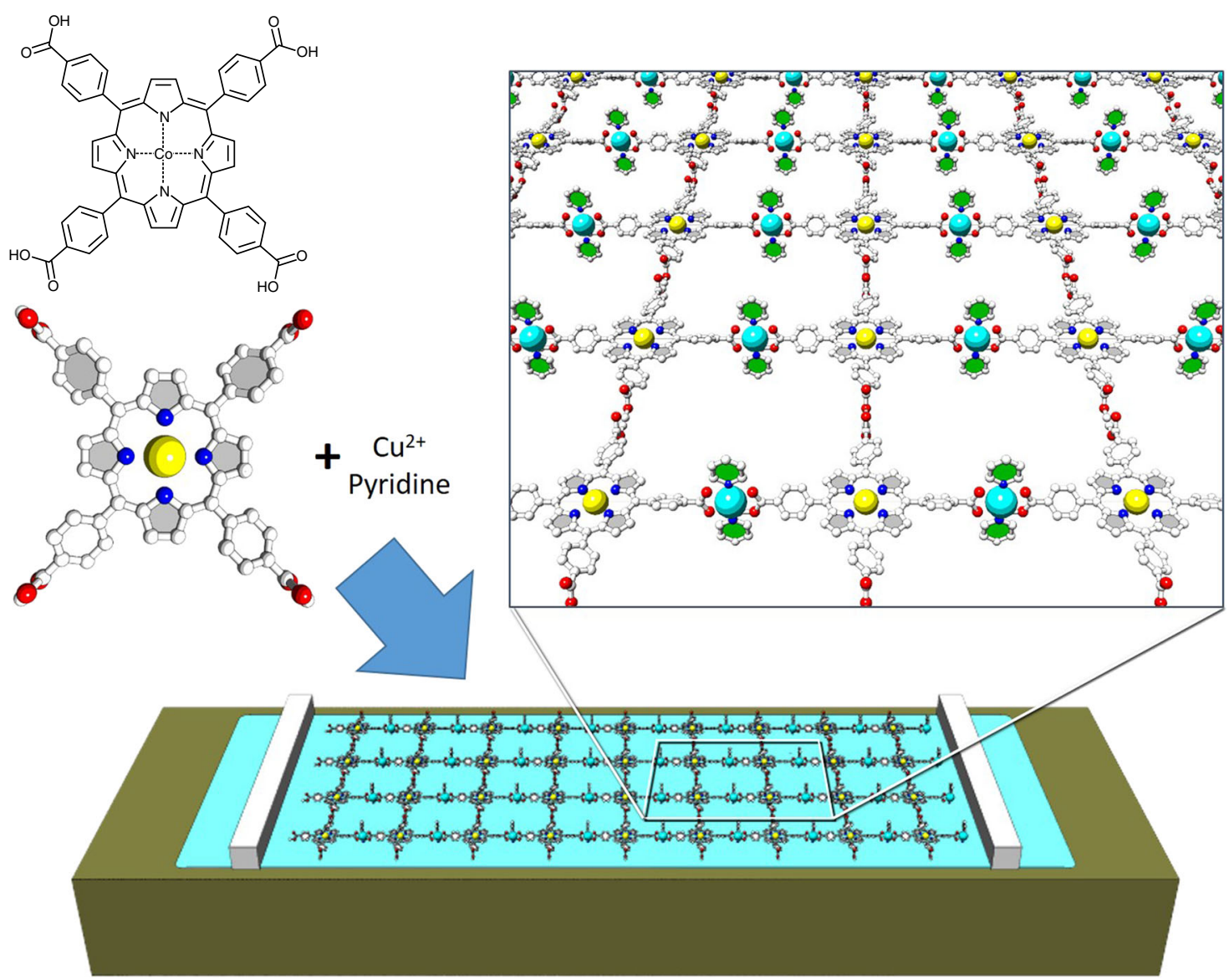

Fig. 7 Two-dimensional assembly of metal-organic framework structures consisting of metalloporphyrin building units

up to $20 \mathrm{~cm}^{2} \mathrm{~V}^{-1} \mathrm{~s}^{-149}$. Nanoarchitectonics could become one of the most indispensable technologies in molecular electronics, constituting a key step towards replacing silicon-based devices for logic applications.

\section{D network materials}

Instead of the well-packed assembly of organic molecules, the formation of network structures in two dimensions via molecular interactions such as hydrogen bonding and metal coordination is a good strategy to obtain another type of soft 2D nanoarchitectonic architecture. Because the efficiency of hydrogen bonding is greatly enhanced at the air-water interface compared with in aqueous solution ${ }^{50,51}$, an air-water interface is the appropriate platform to form hydrogen-bond-based supramolecular polymers with a $2 \mathrm{D}$ geometry. As 


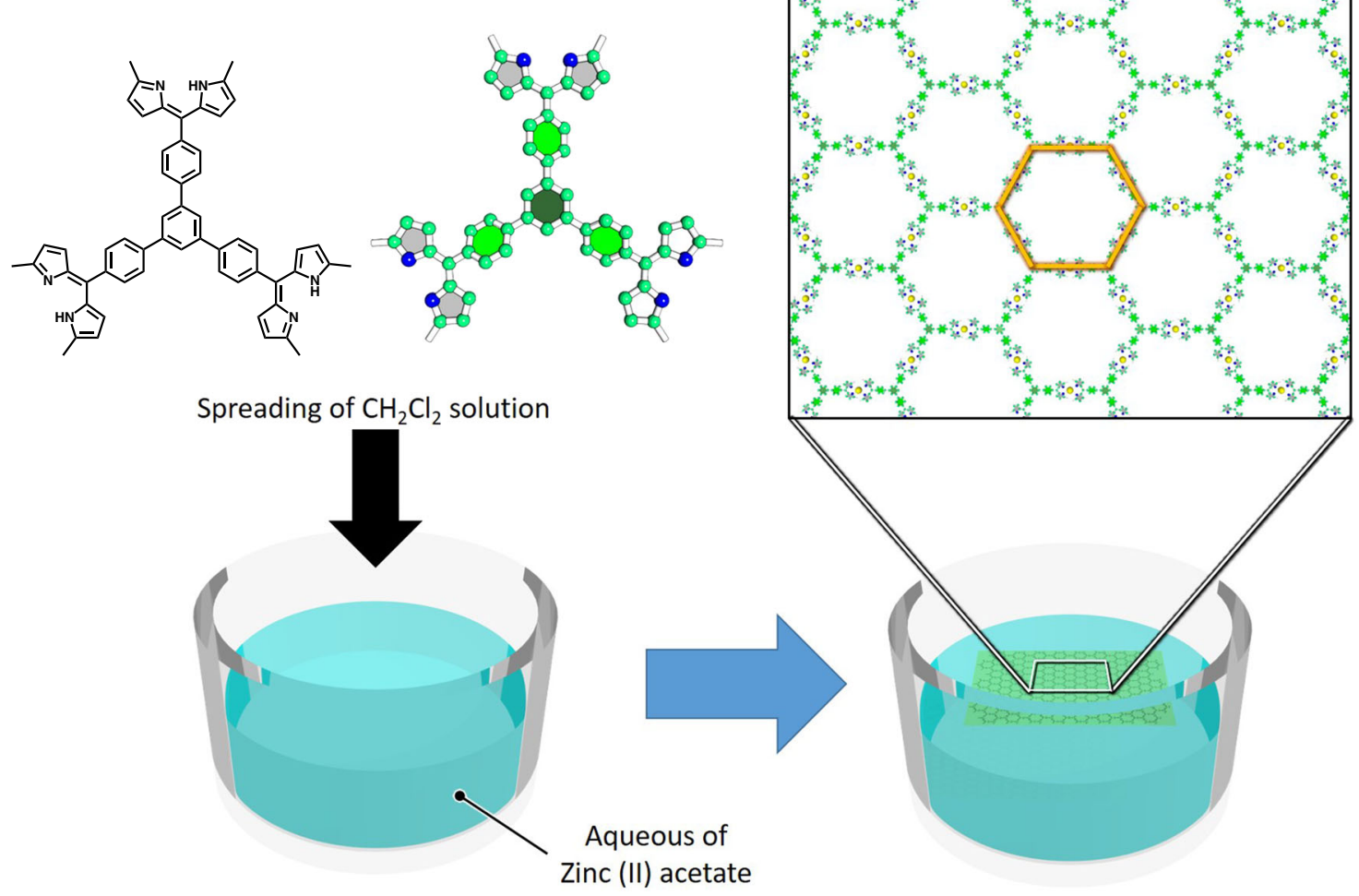

Fig. 8 Photofunctional 2D sheets of metal-organic frameworks through bis(dipyrrinato)zinc(II) bridges using a three-direction-branched organic ligand with three dipyrrinato-functional groups at the air-water interface

illustrated in Fig. 5, dialkylmelamine amphiphiles at the air-water interface spontaneously form hydrogenbonding pairs with aqueous barbituric acid, resulting in a linearly extended supramolecular polymer chain ${ }^{52}$. The use of a branched amphiphile with three melamine rings and six alkyl chains as a monolayer component leads to the formation of a soft hydrogen-bonded network in two dimensions ${ }^{53}$. According to molecular models, it can be expected that two melamine rings of one amphiphile molecule unit form a linear extension of a hydrogenbonded supramolecular polymer chain, and the remaining melamine ring contributes to the formation of an additional hydrogen-bonded chain, resulting in the parallel alignment of supramolecular polymer chains.

As a 2D network structure including metal bonding, Talham and co-workers ${ }^{54}$ demonstrated the formation of a 2D coordinate covalent square grid network at the air-water interface. For this, an amphiphilic pentacyanoferrate complex, bis(tetramethylammonium)pentacyano (4-(didodecylamino)-pyridine)ferrate(III), was used as a monolayer-forming component (Fig. 6). A square grid NiFe cyanide $2 \mathrm{D}$ network structure with $90^{\circ}$ bond angles around the starting iron cyanide complex was formed with the addition of $\mathrm{NaCl}$ and subsequent injection of a $\mathrm{Ni}\left(\mathrm{NO}_{3}\right)_{2}$ solution. For formation of the square grid network, $2 \mathrm{D}$ condensation was initiated immediately after injection of the $\mathrm{Ni}(\mathrm{II})$ ion solution. Various structural characterizations revealed that the formed 2D structure is uniform over an average domain size of $36 \mathrm{~nm}^{2}$. The ferromagnetically ordered state with significant dependency on the orientation of the square grid with respect to the field was probed through magnetic measurements. The condensation reaction at the $2 \mathrm{D}$ air-water interface using the amphiphilic dialkylaminopyridine ligand specifically provides a 2D squire grid structure with particular magnetic properties. In fact, a similar reaction in the 3D solution phase only gave amorphous products

Metal-organic frameworks (or coordination polymers) with well-defined nanostructures constructed from specific coordination between organic ligands and metal components have attracted attention due to their interesting structures and potential applications ${ }^{55-57}$. Converting metal-organic framework motifs into 2D structures has also been recently investigated. Makiura et al. $^{58}$ reported the fabrication of Langmuir-Blodgett (LB) films and the layer-by-layer (LbL) assembly of metalorganic framework structures consisting of metalloporphyrin building units (Fig. 7). A chloroform/methanol solution of 5,10,15,20-tetrakis(4-carboxyphenyl)porphyrinato-cobalt(II) and pyridine was spread on an aqueous subphase containing $\mathrm{Cu}^{2+}$. The formed Langmuir monolayer sheets of the metal-organic framework motif 


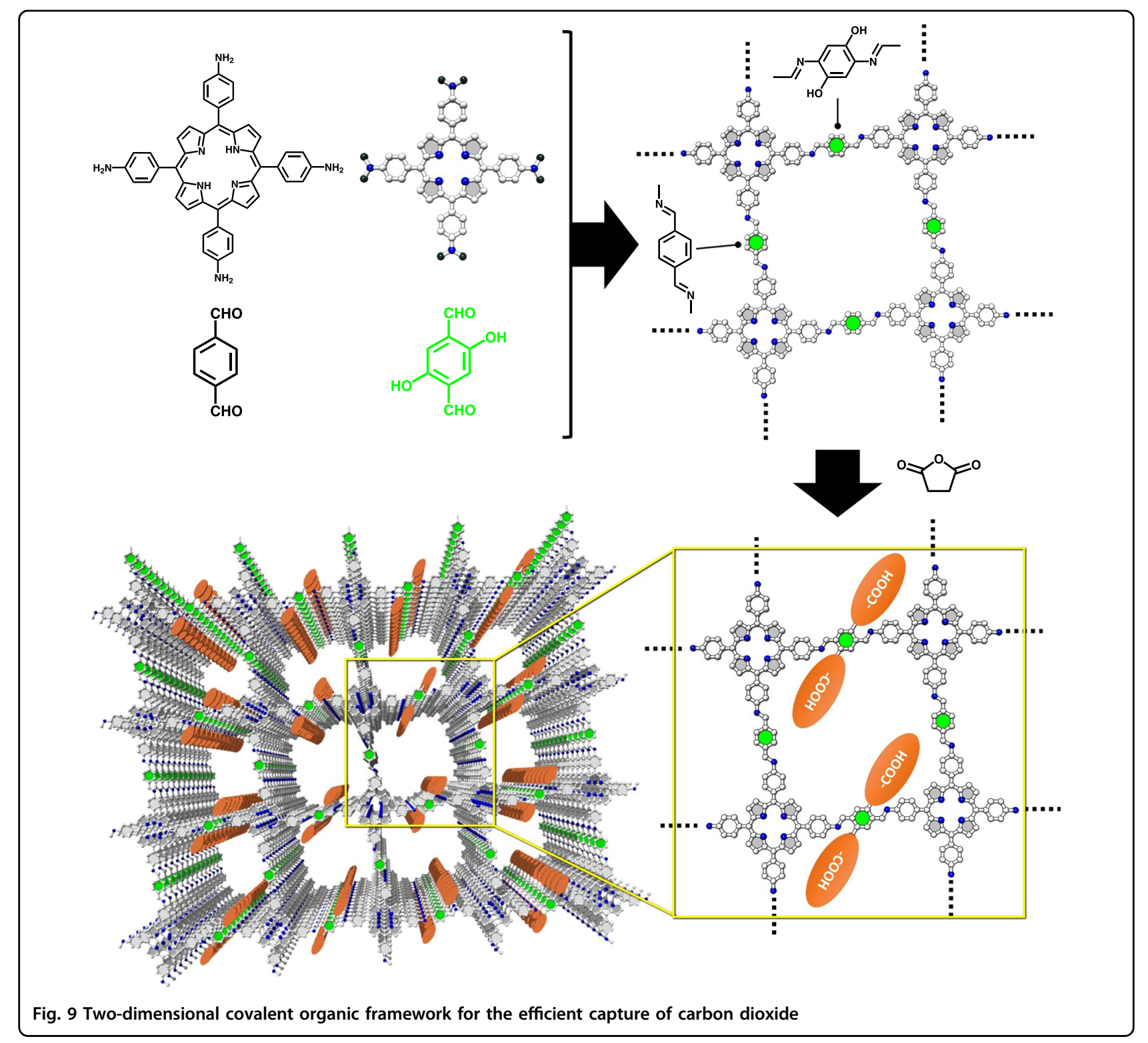

were deposited in a LbL fashion with intermediate rinsing and drying processes. Neighboring metal-organic framework sheets were stacked with $1 / 4$ unit cell shifts with the aid of $\pi-\pi$ interactions between pyridine units coordinating axially to the paddle wheels. Such metal-organic framework structures are supposed to have gate openingclosing properties upon gas adsorption. Therefore, the fabrication of thin films of such structures would be attractive to make a connection between sensing materials and device surfaces. In addition, heterogeneous layering with various $2 \mathrm{D}$ metal-organic frameworks would be useful for well-designed systems for materials separation, storage, various devices, and biological applications.

Sakamoto et al. ${ }^{59,60}$ demonstrated the fabrication of photofunctional 2D sheets of metal-organic frameworks through bis(dipyrrinato)zinc(II) bridges using a threedirection-branched organic ligand with three dipyrrinatofunctional groups at the air-water interface (Fig. 8). Although the formation of a metal-organic framework at liquid-liquid interface through contacting dipyrrin ligand and zinc(II) ions from different solutions usually provides multi-layered nanosheet structures, exposing an organic solution of three-direction-branched organic ligand from the air phase to the aqueous phase produces single-layer or few-layer nanosheets with domain sizes of $>10 \mu \mathrm{m}$, which can be transferred to various substrates by the Langmuir-Schăfer method. The bis(dipyrrinato)zinc(II) complex also shows photosensitive properties with a strong absorption in the visible and near-infrared region. A sensor composed of multi-layered films of bis 


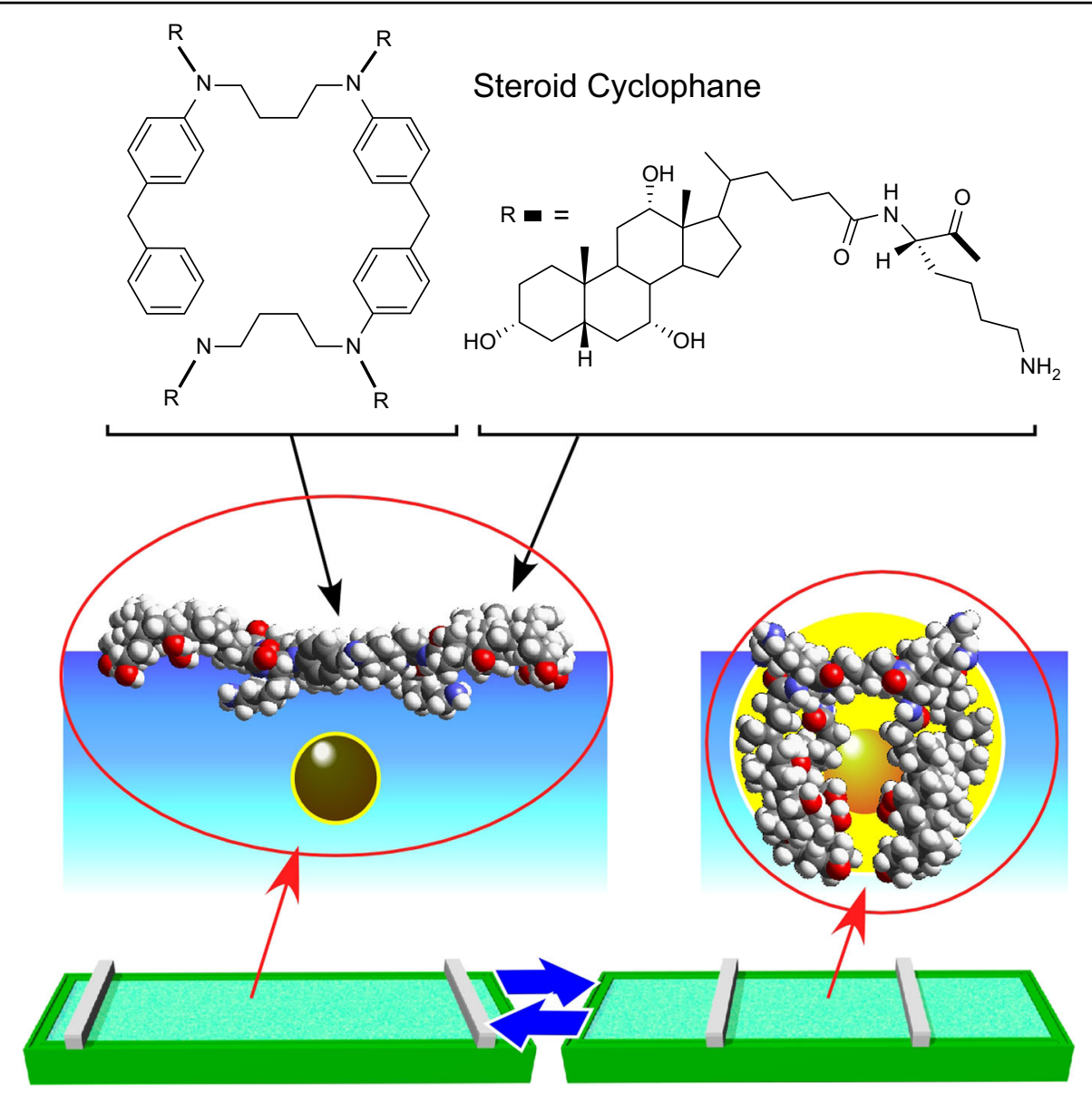

Fig. 10 Catching and releasing a particular guest molecule upon macroscopic lateral compression and expansion of the monolayer of the molecular machine (steroid cyclophane) at the air-water interface

(dipyrrinato)zinc(II)-based metal-organic framework nanosheets on a transparent $\mathrm{SnO}_{2}$ working electrode in a three-electrode system in the presence of triethanolamine as a sacrificial electron donor showed anodic currents only upon irradiation of $500 \mathrm{~nm}$ light. As demonstrated in the above-mentioned example, various functional groups with desired properties can be included in $2 \mathrm{D}$ thin films through coordination-based nanoarchitectonics in 2D geometries.

Instead of using metal-ligand coordination, covalentbond-based network structures can be synthesized, as shown in research examples of covalent organic frameworks. For example, Jiang and co-workers ${ }^{61}$ recently reported the fabrication and functionalization of a $2 \mathrm{D}$ covalent organic framework for the efficient capture of carbon dioxide (Fig. 9). In the first step, a conventional 2D covalent organic framework based on porphyrin with imine linkages at the vertices and phenol groups on the side moieties is prepared. Carboxylic groups can be introduced into the framework structure through the ring-opening reaction of succinic anhydride with the phenol groups. Within the porous structure of the stacked covalent organic frame, the introduced carboxylate groups locate at the surfaces of the pores. The density of carboxylate groups can be tuned by adjusting the amount of phenol groups in the original covalent organic framework structure. According to experiments with gases, the functionalized porous materials showed selective adsorption toward $\mathrm{CO}_{2}$ over $\mathrm{N}_{2}$. Similar modifications of the inside surfaces of covalent organic frameworks with various functional groups are possible through conventional organic chemistry. This approach would be useful for the design of materials for high-performance gas storage and separation.

\section{D control of molecular machines and receptors}

Soft 2D nanoarchitectonics is not limited to the preparation of functional materials and the fabrication of device structures in two dimensions. Dynamic processes, such as self-assembly, molecular recognition, and molecular manipulation, are other attractive targets in soft $2 \mathrm{D}$ systems $^{62}$. Unlike 3D media, restricted molecular motions 


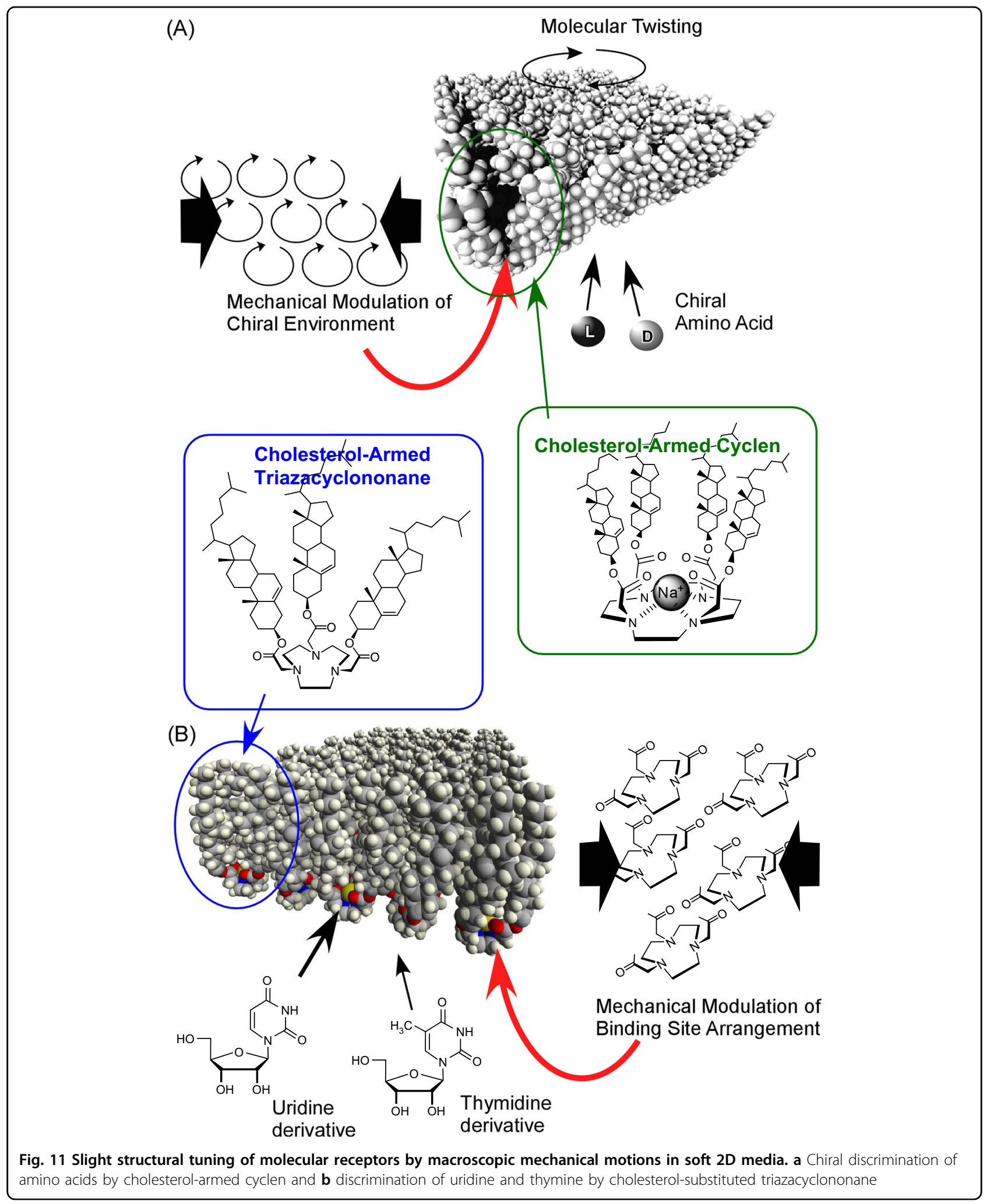

within confined 2D spaces often result in well-defined orientation, ordering, selection, and efficiency in these processes. It is known that binding constants for molecular recognition are incredibly enhanced at the air-water interface compared with those observed in bulk aqueous media. For example, binding constant between 
(A) One Stable State

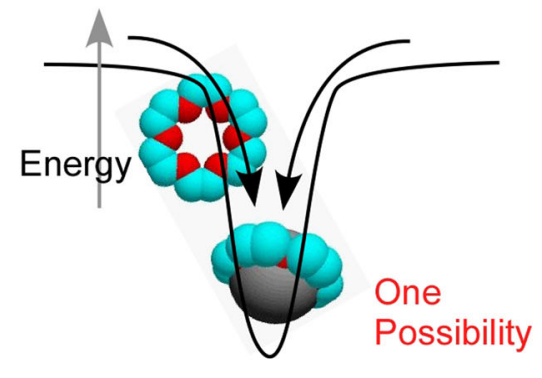

(B) Switching Mode

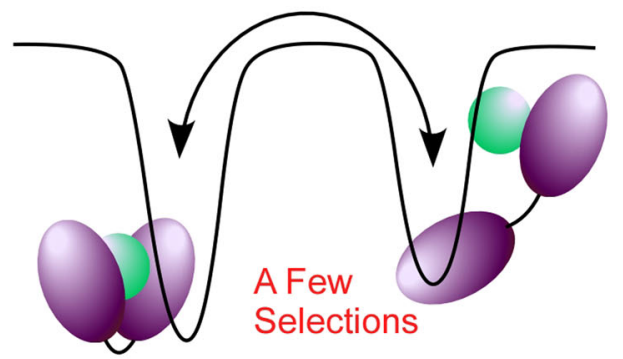

\section{(C) Tuning Mode}

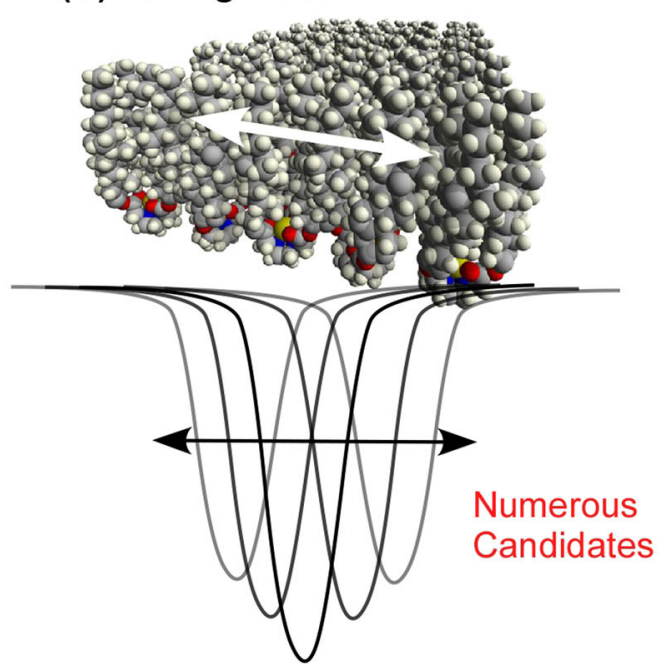

Fig. 12 Categories of molecular recognition modes in the history of supramolecular chemistry. a One stable state of a host-guest complex, b switching mode, and c tuning mode

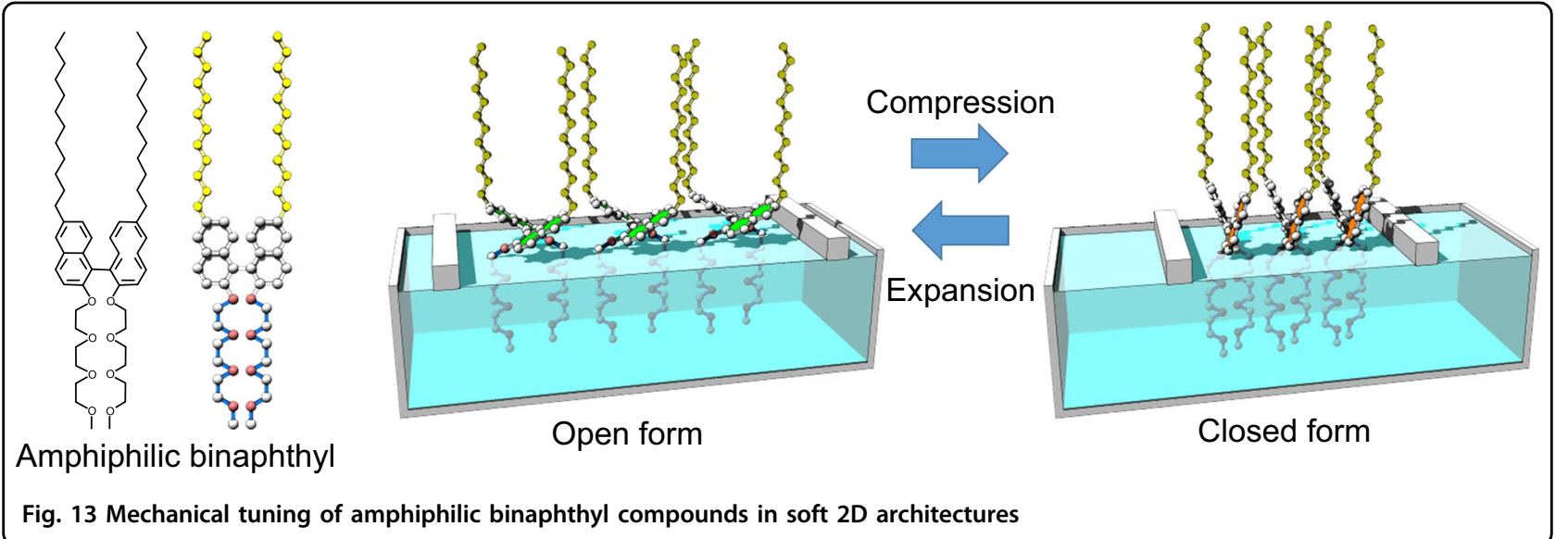

guanidinium and phosphate at the 2D air-water interface are in the range of $10^{6}$ and $10^{7} \mathrm{M}^{-1}$, while the corresponding constant is only $1.4 \mathrm{M}^{-1}$ in the $3 \mathrm{D}$ aqueous phase $^{63,64}$. According to theoretical simulations based on a quantum chemical approach, heterogenic dielectric features at the 2D interface of media significantly affect the nature of molecular interactions, which are fundamental aspects of supramolecular chemistry. 


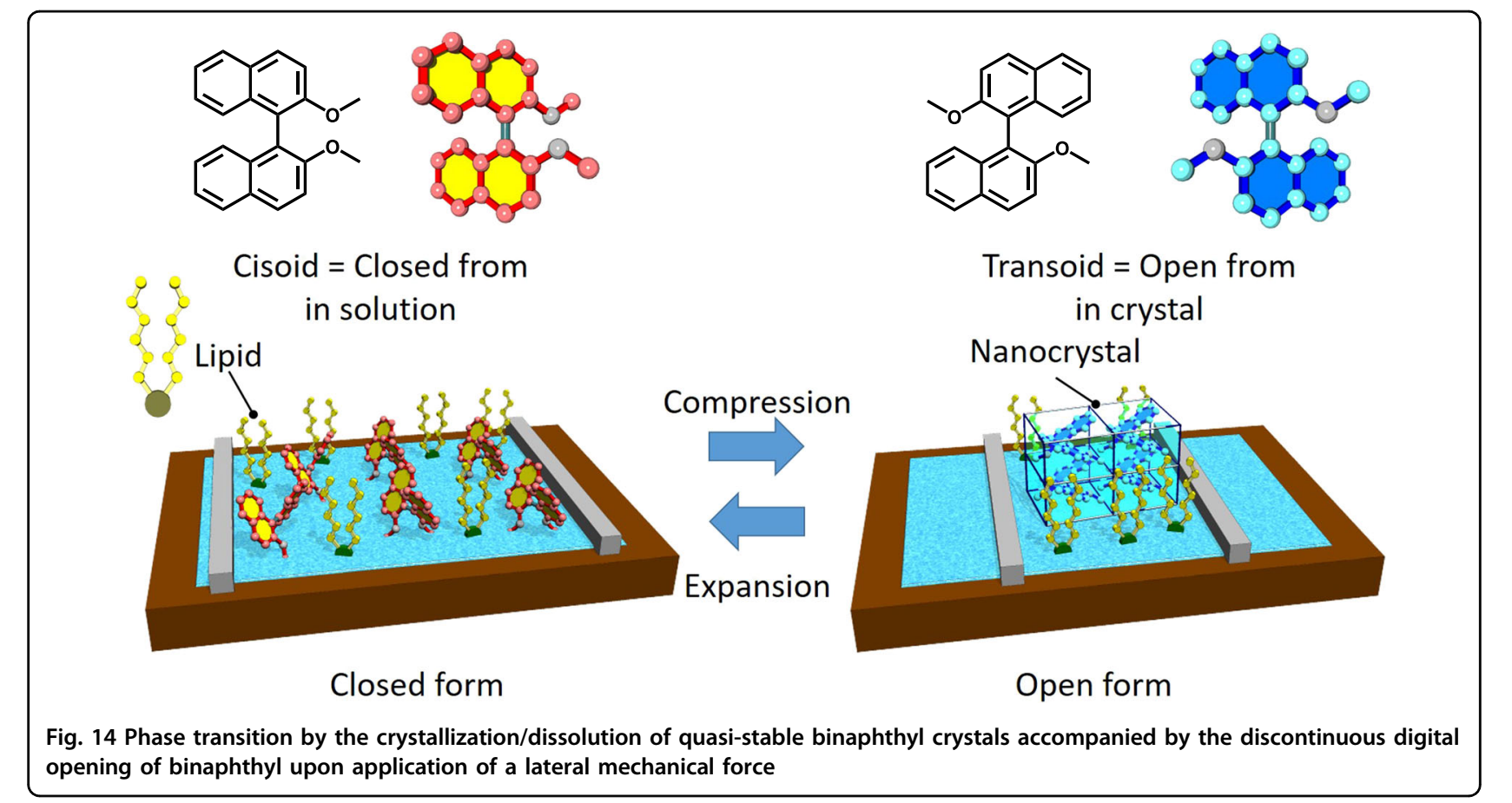

As they are not limited to confinement requirements and heterogenic dielectric features, dynamic interfaces have specific advantages in coupling between extremely different-sized events, such as centimeter- or meter-level macroscopic mechanical motions and nanometer-level molecular functions. Dynamic 2D media, such as air-water interfaces, have lateral sizes on the macroscale, but their thickness is confined within the nanoscale. Therefore, dynamic 2D interfaces are appropriate media for coupling macroscopic mechanical stimuli and molecular functions ${ }^{65}$. This intrinsic nature of dynamic $2 \mathrm{D}$ media enables us to accomplish incredible actions, such as control of molecular machines and tuning of molecular receptors, by hand-motion-like macroscopic mechanical motions ${ }^{66}$. For example, the molecular machine (steroid cyclophane) system exemplified in Fig. 10 is capable of catching and releasing a particular guest molecule upon macroscopic lateral compression and expansion of its monolayer formed at the air-water interface ${ }^{67,68}$. The control of molecular machines through nanotechnological actions can be accomplished by human-level actions, such as hand motions, which is defined as hand-operated nanotechnology.

Controllable molecular motions are not limited to large apparent conformational changes, such as the cavity formation and opening described in the previous example. Slight structural tuning of molecular receptors can be achieved by macroscopic mechanical motions of soft 2D media. Two examples of molecular receptors are illustrated in Fig. 11: (A) cholesterol-armed cyclen ${ }^{69}$ and (B) cholesterol-substituted triazacyclononane ${ }^{70}$. In the former case, the helical structures of the molecular receptors, which contain four chiral cholesteryl residues, are influenced by changes in their packing states by external conditions, such as mechanical pressure. Upon embedding the cholesterol-armed cyclen receptors at the air-water interface, their guest accommodation space can be tuned by the application of lateral pressure to their monolayer, accompanied by a shift in their diastereomeric stability upon binding of chiral guests from the aqueous subphase. For example, the binding constant of D-valine to the cholesterol-armed cyclen monolayer exceeds that for the $\mathrm{L}$ isomer at lower surface pressure, showing $\mathrm{D}$ preferential binding, but the binding constant to L-valine becomes larger than that to D-valine at increased surface pressure. The observed inversion of the enantiomeric selectivity toward amino acids can be achieved by tuning the receptor structures through macroscopic mechanical motions. This receptor tuning concept can be applied to realize the efficient differentiation between aqueous thymine and uracil derivatives by using mechanically adjustable cholesterol-substituted triazacyclononane receptors at the air-water interface. Under the selected conditions, the binding constant for uracil recognition becomes 60-70 times larger than that for thymine, although these nucleic acid bases, which have a structural difference of only one methyl group, cannot be distinguished by DNA or RNA.

Molecular recognition in the above-mentioned examples utilized the fundamental structural flexibility of organic compounds. This way of thinking is different from well-known molecular recognition techniques that rely on 


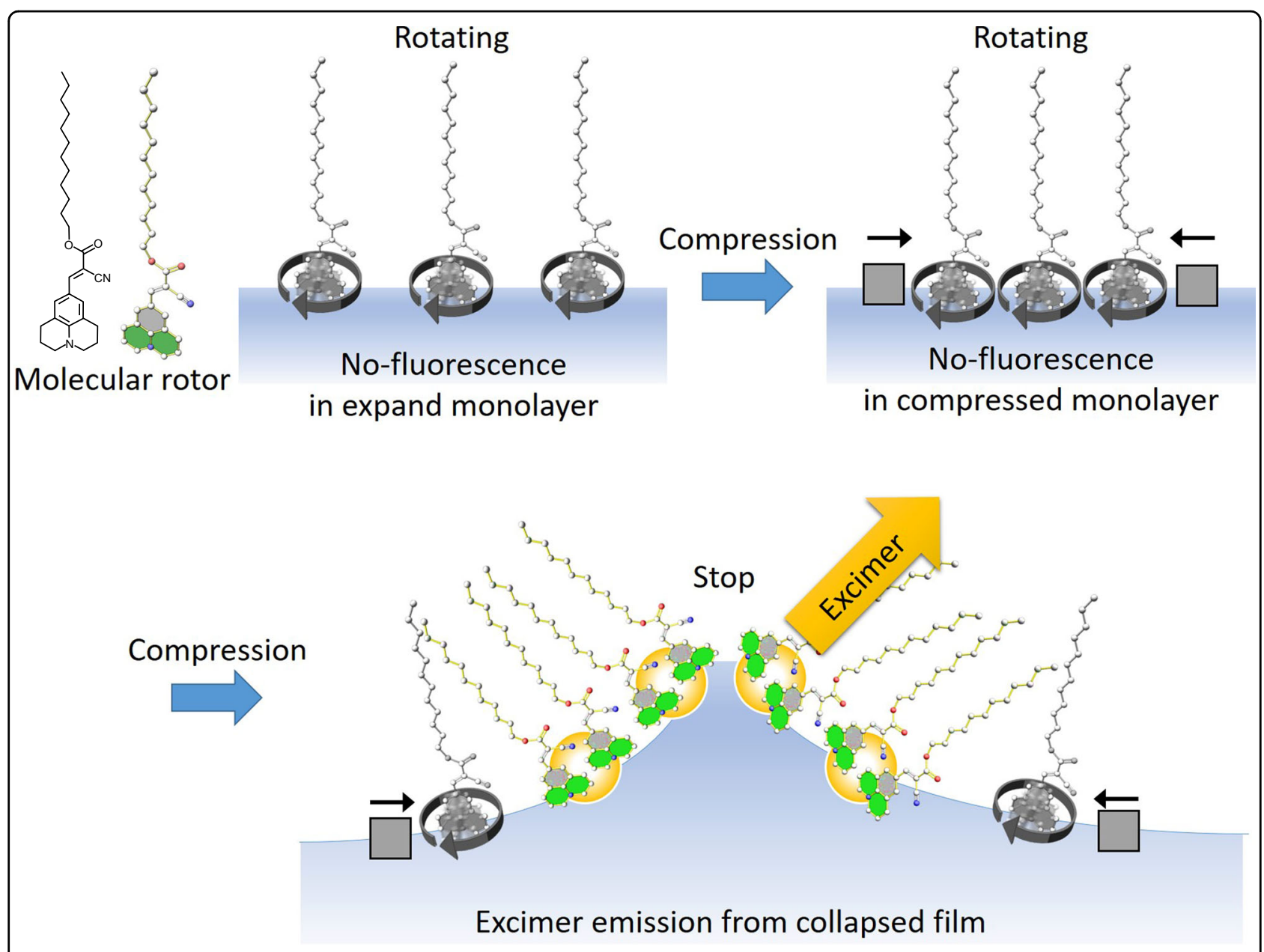

Fig. 15 Intramolecular motions of amphiphilic molecular rotors confined within two dimensions

information from single-crystalline structures and computational optimization. Figure 12 summarizes categories of molecular recognition modes in the history of supramolecular chemistry ${ }^{71,72}$. The most fundamental mode is based on a single stable state of a host-guest complex, as seen for molecular recognition with conventional artificial and biological receptors, such as crown ethers, cyclodextrins, and antibiotics (Fig. 12a). A switching mode was first demonstrated by Shinkai et al $^{73}$ in their pioneering work on photoswitchable azo-benzene hosts and expanded to include many kinds of molecular recognition systems. This switching mode creates multiple (two or three or four, etc.) stable states, and the recognition behavior can switch among these states (Fig. 12b). In contrast, the tuning concept explained above can be regarded as an emerging mode of molecular recognition (Fig. 12c). Through confining molecular receptors in soft 2D nanoarchitectures, the molecular states and energy states of molecules can be continuously shifted through macroscopic lateral mechanical motions. This mode imparts a countless number of receptor conformers as candidates for desired recognitions and functions. We can take the most desirable host structures from this continuous tuning process. This tuning mode is thus a novel mode of molecular recognition.

The mechanical tuning of molecules in soft 2D architectures has been systematically investigated using amphiphilic binaphthyl compounds, because changes in the dihedral angle of the binaphthyl moiety can be easily monitored and theoretically simulated ${ }^{74}$. As illustrated in Fig. 13, the monolayer of the amphiphilic binaphthyl molecules is continuously compressed at the air-water interface and has been subjected to detailed structural analyses and theoretical calculations. At this soft $2 \mathrm{D}$ medium, the application of small mechanical energy $(<1$ $\mathrm{kcal} \mathrm{mol}^{-1}$ ) is ensured, accompanied by a large variation in molecular area $(>50 \%)$. The combination of several approaches, including experimental measurements of circular dichroism, thermodynamic energy calculations, single-molecule simulations, and molecular dynamics 


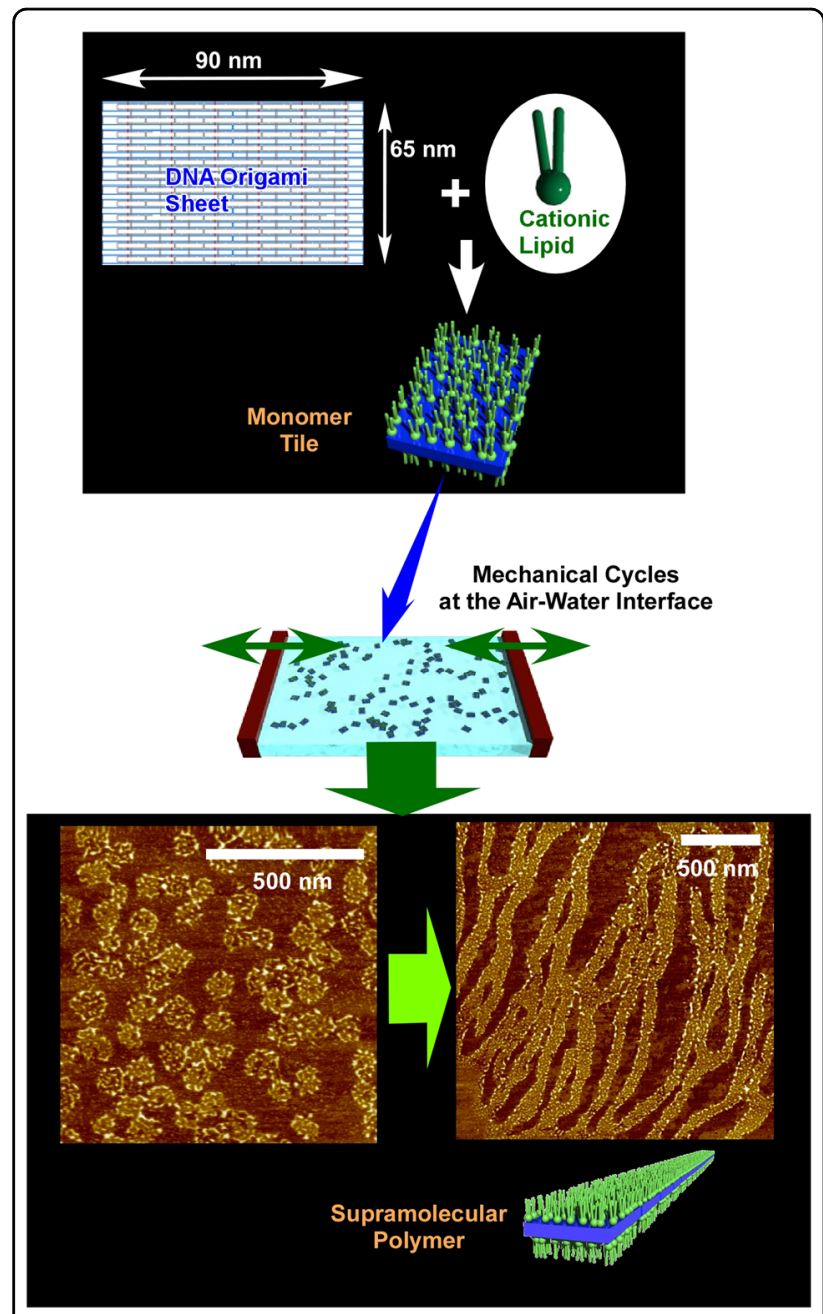

Fig. 16 Mechanically driven supramolecular polymerization of DNA origami into a particular 1D direction

simulations, has revealed various facts concerning molecular tuning at the 2D interface of media. Mechanical energy can be proportionally converted to torsional energy in the binaphthyl molecule. The global average torsion angle of the binaphthyl molecule can be continuously shifted. Therefore, this molecular tuning is regarded as an analogous control of molecular conformation, possibly with pN-level forces.

The modulation of molecular components induces changes in the molecular control from analog mode to digital mode ${ }^{75}$. When rather simple binaphthyl molecules are mixed in lipid matrices, a phase transition involving the crystallization/dissolution of quasi-stable binaphthyl crystals accompanied by the discontinuous digital opening of the binaphthyl moiety can be regulated by the application of a lateral mechanical force (Fig. 14). These changes are reversible and repeatable. These examples represent a step forward in the development of mechanical tools of molecular machines with continuous and reversible motion under an external force. In these attempts, kinetic and thermodynamic investigations as well as theoretical simulations are necessary to construct 2D systems to accurately control molecular machines.

Very recently, Nakanishi and co-workers ${ }^{76}$ investigated the intramolecular motions of amphiphilic molecular rotors confined within two dimensions and further compared their behaviors with those in three dimensions (Fig. 15). The studied molecular rotors were 9-(2-carboxy2-cyanovinyl)julolidine derivatives with fluorescence properties and twisted intramolecular charge-transfer capability. The suppression of their intramolecular rotation can be monitored by an increase in their fluorescence intensity. The in situ fluorescence measurement of the molecular rotor monolayer upon compression revealed that the intramolecular rotation was not suppressed even in condensed monolayers. In the $2 \mathrm{D}$ condensed phase with molecular alignment, the cross-section of the hydrophobic region of the molecular rotor ensures free rotation of the rotor. This molecular rotation was maintained in transferred LB films. In contrast, expanding the monolayers into three dimensions inhibits molecular rotation and promotes excimer formation. These examples indicate that well-considered molecular ordering is crucial to maintain free spaces for molecular motion to preserve molecular rotor motion in high-density states. Two-dimensional nanoarchitectonic design would be advantageous to operate molecular machines within condensed phases.

\section{Perspectives}

In this short review, we exemplified various approaches of nanoarchitectonics within two dimensions using soft components. The appropriate design of component molecules leads to the formation of defect-less 2D layers over incredibly large areas, high-performance organic devices, 2D networks with well-defined structures, and the free rotation of molecular machines within a $2 \mathrm{D}$ condensed phase. Molecular orientation, ordering, and mutual interaction are also important factors for the observed properties and functions, as well as the molecular designs themselves. As the second focus of soft $2 \mathrm{D}$ nanoarchitectonics, the efficient connection of macroscopic forces and actions with molecular functions is emphasized to play another role in soft $2 \mathrm{D}$ interfacial environments in this short review. Molecular machines and molecular receptors can be tuned by hand-motionlike macroscopic mechanical motions. Molecules in soft 2D media can be mechanically handled with incredibly small forces, possibly on the pN level.

Research targets of soft 2D nanoarchitectonics are not limited to organic matter, such as their 2D structures, networks, devices, molecular machines, and receptors. 


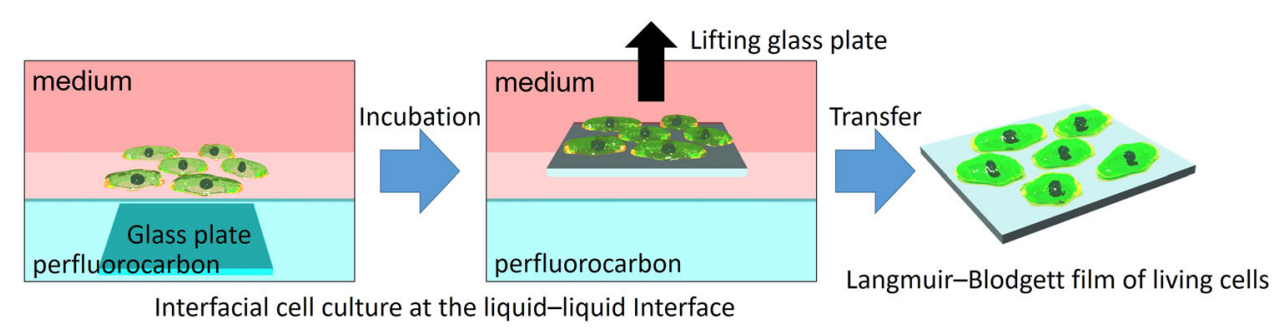

Fig. 17 Cell cultures at completely soft liquid-liquid interfaces to realize a Langmuir-Blodgett film of living cells

This concept could be expanded to bio-related systems, because structural control and tuning with very weak forces through certain harmonization with turbulence from the surrounding environment are crucial for the regulation of biological systems. Several attempts have been made to control bio-systems at the air-water interface, as a typical soft 2D nanoarchitectonic medium. Figure 16 illustrates a Langmuir monolayer of DNA origami pieces and their mechanically driven supramolecular polymerization into a particular $1 \mathrm{D}$ direction ${ }^{77}$. Although programs of DNA sequences can only promote squareshaped unit formation, dynamic movements upon monolayer compression and expansion induce further alignment of individual pieces into quite long 1D organizations that cannot be obtained by DNA programing alone.

Very recently, cell cultures have been investigated at completely soft liquid-liquid interfaces (Fig. 17) ${ }^{78}$. This is the first example of the preparation of an LB-type film of living cells. Differentiation of the tested cells was efficiently suppressed, likely because of the ultimate softness of the scaffold environment, while cell differentiation was promoted on a rigid surface with aligned fullerene assemblies $^{79,80}$. These examples indicate that 2D nanoarchitectonics is a powerful strategy for the intentional organization and functional control of soft biomaterials, including living cells. In addition to materials design, interfacial environments themselves can be powerful media for cell regulation. More sophisticated investigations using stem cells would lead to novel methodologies for cell technology and tissue engineering.

The unit components of living creatures, such as proteins and DNA, are simply materials and molecules, but their logical organization converts non-living materials to active living presences. The boarder between non-living matter and living entities is likely located at regions scaling from nanometers to submicrometers, which is the working scale of nanoarchitectonic approaches. Therefore, nanoarchitectonics can play an important role as a navigator between materials science to life science ${ }^{81}$. In particular, nanoarchitectonic designs confined within soft 2D media are very similar to the rational organization of functional molecules within cell membranes. Soft 2D nanoarchitectonics with biological components would create a new paradigm for materials development in biotechnology, biomedical science, biodevices, and biolike efficient energy conversions. As seen in the previous pioneering works, thermodynamics and kinetics, with the requirement of a $2 \mathrm{D}$ confined space ${ }^{82,83}$ and the dynamic evolution of soft building blocks through spatiotemporal organization $^{84,85}$, create distinct features in their functions that cannot be achieved in $3 \mathrm{D}$ systems ${ }^{86}$.

\section{Acknowledgements}

This study was partially supported by JSPS KAKENHI Grant Number JP16H06518 (Coordination Asymmetry) and CREST JST Grant Number JPMJCR1665.

\section{Author details}

'World Premier International (WPI) Research Center for Materials Nanoarchitectonics (MANA), National Institute for Materials Science (NIMS), 1-1 Namiki, Tsukuba 305-0044, Japan. ${ }^{2}$ Department of Advanced Materials Science, Graduate School of Frontier Sciences, The University of Tokyo, 5-1-5

Kahiwanoha, Kashiwa, Chiba 277-8561, Japan. ${ }^{3}$ JST, PRESTO, 4-1-8 Honcho, Kawaguchi, Saitama 332-0012, Japan

\section{Conflict of interest}

The authors declare that they have no conflict of interest.

\section{Publisher's note}

Springer Nature remains neutral with regard to jurisdictional claims in published maps and institutional affiliations.

Received: 19 October 2017 Revised: 9 December 2017 Accepted: 4 February 2018.

Published online: 6 April 2018

\footnotetext{
References

1. Bauer, I. \& Knölker, H.-J. Iron catalysis in organic synthesis. Chem. Rev. 115, 3170-3387 (2015).

2. Yamashita, M. The organometallic chemistry of boron-containing pincer ligands based on diazaboroles and carboranes. Bull. Chem. Soc. Jpn. 89, 269-281 (2016).

3. Skubi, K. L., Blum, T. R. \& Yoon, T. P. Dual catalysis strategies in photochemical synthesis. Chem. Rev. 116, 10035-10074 (2016).

4. Frihed, T. G. \& Fürstner, A. Progress in the trans-reduction and transhydrometalation of internal alkynes. Appl. Nat. Product Synth. Bull. Chem. Soc. Jpn. 89, 135-160 (2016).

5. Diner, C. \& Szabó, K. J. Recent advances in the preparation and application of allylboron species in organic synthesis. J. Am. Chem. Soc. 139, 2-14 (2017).

6. Yamaguchi, J. \& Itami, K. Toward an ideal synthesis of (bio) molecules through direct arene assembling reactions. Bull. Chem. Soc. Jpn. 90, 367-383 (2017).
} 
7. Mitsui, C. et al. Oxygen- and sulfur-bridged bianthracene V-shaped organic semiconductors. Bull. Chem. Soc. Jpn. 90, 931-938 (2017).

8. Ramanathan, M. et al. Amphiphile nanoarchitectonics: from basic physical chemistry to advanced applications. Phys. Chem. Chem. Phys. 15, 10580-10611 (2013).

9. Ariga, K. et al. Enzyme nanoarchitectonics: organization and device application. Chem. Soc. Rev. 42, 6322-6345 (2013).

10. Yang, Z., Liu, H. \& Liu, D. Spatial regulation of synthetic and biological nanoparticles by DNA nanotechnology. NPG Asia Mater. 7, e161 (2015).

11. Richardson, J. J., Björnmalm, M. \& Caruso, F. Technology-driven layer-by-layer assembly of nanofilms. Science 348, aaa2491 (2015).

12. Xu, B., Zhou, G. \& Wang, X. Rational synthesis and the structure-property relationships of nanoheterostructures: a combinative study of experiments and theory. NPG Asia Mater. 7, e164 (2015).

13. Pandian, G. N. \& Sugiyama, H. Nature-inspired design of smart biomaterials using the chemical biology of nucleic acids. Bull. Chem. Soc. Jpn. 89, 843-868 (2016).

14. Tobe, Y., Tahara, K. \& De Feyter, S. Adaptive building blocks consisting of rigid triangular core and flexible alkoxy chains for self-assembly at liquid/solid interfaces. Bull. Chem. Soc. Jpn. 89, 1277-1306 (2016).

15. Komiyama, M., Yoshimoto, K., Sisido, M. \& Ariga, K. Chemistry can make strict and fuzzy controls for bio-systems: DNA nanoarchitectonics and cellmacromolecular nanoarchitectonics. Bull. Chem. Soc. Jpn. 90, 967-1004 (2017).

16. Mishra, P. et al. Current-driven supramolecular motor with in-situ surface chira directionality switching. Nano Lett. 15, 4793-4798 (2015).

17. Shirai, Y. et al. Driving nanocars and nanomachines at interfaces: from concept of nanoarchitectonics to actual use in world wide race and hand operation. Jpn. J. Appl. Phys. 55, 1102A2 (2016).

18. Qian, D., Ma, C., More, K. I., Meng, Y. S. \& Chi, M. Advanced analytical electron microscopy for lithium-ion batteries. NPG Asia Mater. 7, e193 (2015).

19. Vance, M. E. et al. Nanotechnology in the real world: redeveloping the nanomaterial consumer products inventory. Beilstein J. Nanotechnol. 6, 1769-1780 (2015).

20. Kiguchi, M. \& Fujii, S. Governing the metal-molecule interface: towards new functionality in single-molecule junctions. Bull. Chem. Soc. Jpn. 90, 1-11 (2017).

21. Ariga, K., Ji, Q., Hill, J. P., Bando, Y. \& Aono, M. Forming nanomaterials as layered functional structures towards materials nanoarchitectonics. NPG Asia Mater. 4 e17 (2012).

22. Ariga, K. \& Aono, M. Nanoarchitectonics. Jpn. J. Appl. Phys. 55, 1102 A6 (2016).

23. Ariga, K. et al. Inorganic nanoarchitectonics for biological applications. Chem. Mater. 24, 728-737 (2012).

24. Nakanishi, W. et al. Bioactive nanocarbon assemblies: nanoarchitectonics and applications. Nano Today 9, 378-394 (2014).

25. Ishihara, S. et al. Porphyrin-based sensor nanoarchitectonics in diverse physical detection modes. Phys. Chem. Chem. Phys. 16, 9713-9746 (2014).

26. Rydzek, G. et al. Electrochemical nanoarchitectonics and layer-by-layer assembly: from basics to future. Nano Today 10, 138-167 (2015).

27. Ariga, K., Li, J., Fei, J., Ji, Q. \& Hill, J. P. Nanoarchitectonics for dynamic functional materials from atomic/molecular-level manipulation to macroscopic action. Adv. Mater. 28, 1251-1286 (2016).

28. Ariga, K., Minami, K. \& Shrestha, L. K. Nanoarchitectonics for carbon-materialbased sensors. Analyst 141, 2629-2638 (2016).

29. Abe, H., Liu, J. \& Ariga, K. Catalytic nanoarchitectonics for environmentallycompatible energy generation. Mater. Today 19, 12-18 (2016).

30. Ariga, K., Li, M., Richards, G. J. \& Hill, J. P. Nanoarchitectonics: a conceptual paradigm for design and synthesis of dimension-controlled functional nanomaterials. J. Nanosci. Nanotechnol. 11, 1-13 (2011).

31. Ariga, K., Ji, Q., Nakanishi, W., Hill, J. \& Aono, M. Nanoarchitectonics: a new materials horizon for nanotechnology. Mater. Horiz. 2, 406-413 (2015).

32. Aono, M. \& Ariga, K. The way to nanoarchitectonics \& the way of nanoarchitectonics. Adv. Mater. 28, 989-992 (2016).

33. Gambhir, S., Jalili, R., Officer, D. L. \& Wallace, G. G. Chemically converted graphene: scalable chemistries to enable processing and fabrication. NPG Asia Mater. 7, e186 (2015).

34. Khan, A. H. et al. Two-dimensional (2D) nanomaterials towards electrochemical nanoarchitectonics in energy-related applications. Bull. Chem. Soc. Jpn. 90 627-648 (2017).

35. Ariga, K., Lee, M. V., Mori, T., Yu, X.-Y. \& Hill, J. P. Two-dimensional nanoarchitectonics based on self-assembly. Adv. Colloid Interf. Sci. 154, 20-29 (2010).

36. Zhuang, X., Mai, Y., Wu, D., Zhang, F. \& Feng, X. Two-dimensional soft nanomaterials: a fascinating world of materials. Adv. Mater. 27, 403-427 (2015).
37. Ariga, $\mathrm{K}$. et al. Challenges and breakthroughs in recent research on selfassembly. Sci. Technol. Adv. Mater. 9, 014109 (2008).

38. Hanabusa, K. \& Suzuki, M. Physical gelation by low-molecular-weight compounds and development of gelators. Bull. Chem. Soc. Jpn. 89, 174-182 (2016)

39. Stang, P. J. \& Olenyuk, B. Self-assembly, symmetry, and molecular architecture: coordination as the motif in the rational design of supramolecular metallacyclic polygons and polyhedra. Acc. Chem. Res. 30, 502-518 (1997).

40. Fiori, G. et al. Electronics based on two-dimensional materials. Nat. Nanotechnol. 9, 768-779 (2014).

41. Hasegawa, T. \& TakeyaJ. J. Organic field-effect transistors using single crystals. Sci. Technol. Adv. Mater. 10, 024314 (2009).

42. Seiki, N. et al. Rational synthesis of organic thin films with exceptional longrange structural integrity. Science $\mathbf{3 4 8}, 1122-1126$ (2015).

43. Takeya, J. et al. In-crystal and surface charge transport of electric-field-induced carriers in organic single-crystal semiconductors. Phys. Rev. Lett. 98, 196804 (2007).

44. Zhang, Y. et al. Probing carrier transport and structure-property relationship of highly ordered organic semiconductors at the two-dimensional limit. Phys. Rev. Lett. 116, 016602 (2016).

45. Diao, Y. et al. Solution coating of large-area organic semiconductor thin films with aligned single-crystalline domains. Nat. Mater. 12, 665-671 (2013).

46. Minemawari, $H$. et al. Inkjet printing of single crystal films. Nature 475, 364-367 (2011).

47. Uno, M. et al. High-yield, highly uniform solution-processed organic transistors integrated into flexible organic circuits. Adv. Electron. Mater. 3, 1600410 (2017).

48. Yamamura, A. et al. Painting integrated complementary logic circuits for single-crystal organic transistors: a demonstration of a digital wireless communication sensing tag. Adv. Electron. Mater. 3, 1600456 (2017).

49. Tsurumi, J. et al. Coexistence of ultra-long spin relaxation time and coherent charge transport in organic single-crystal semiconductors. Nat. Phys. 13, 994-998 (2017).

50. Sakurai, M., Tamagawa, H., Inoue, Y., Ariga, K. \& Kunitake, T. Theoretical study of intermolecular interaction at the lipid-water interface. 1. Quantum chemical analysis using a reaction field theory. J. Phys. Chem. B 101, 4810-4816 (1997).

51. Ariga, K. \& Kunitake, T. Molecular recognition at air-water and related interfaces: complementary hydrogen bonding and multisite interaction. Acc. Chem. Res. 31, 371-378 (1998).

52. Koyano, $\mathrm{H}$. et al. Atomic force microscopic observation of a dialkylmelamine monolayer on barbituric acid. Chem. Commun. 1769-1770 (1996).

53. Koyano, H., Bissel, P., Yoshihara, K., Ariga, K. \& Kunitake, T. Syntheses and interfacial hydrogen-bonded network of hexaalkyl tris(melamine) amphiphiles. Langmuir 13, 5426-5432 (1997).

54. Culp, J. T., Park, J.-H., Stratakis, D., Meisel, M. W. \& Talham, D. R. Supramolecular assembly at interfaces: formation of an extended two-dimensional coordinate covalent square grid network at the air-water interface. J. Am. Chem. Soc. 124, 10083-10090 (2002).

55. Bai, Y. et al. Zr-based metal-organic frameworks: design, synthesis, structure, and applications. Chem. Soc. Rev. 45, 2327-2367 (2016).

56. Cui, Y. et al. Metal-organic frameworks as platforms for functional materials. Acc. Chem. Res. 49, 483-493 (2016).

57. Yamada, T., Sadakiyo, M., Shigematsu, A. \& Kitagawa, H. Proton-conductive metal-organic frameworks. Bull. Chem. Soc. Jpn. 89, 1-10 (2016).

58. Makiura, R. et al. Surface nano-architecture of a metal-organic framework. Nat. Mater. 9, 565-571 (2010).

59. Sakamoto, R. Bottom-up creation of functional low-dimensional materials based on metal complexes. Bull. Chem. Soc. Jpn. 90, 272-278 (2017).

60. Sakamoto, R. et al. A photofunctional bottom-up bis(dipyrrinato)zinc(II) complex nanosheet. Nat. Commun. 6, 6713 (2015).

61. Huang, N., Chen, X., Krishna, R. \& Jiang, D. Two-dimensional covalent organic frameworks for carbon dioxide capture through channel-wall functionalization. Angew. Chem. Int. Ed. 54, 2986-2990 (2015).

62. Ariga, K, Yamauchi, Y., Mori, T. \& Hill, J. P. What can be done with the Langmuir-Blodgett method? Recent developments and its critical role in materials science. Adv. Mater. 25, 6477-6512 (2013).

63. Onda, M., Yoshihara, K., Koyano, H., Ariga, K. \& Kunitake, T. Molecular recognition of nucleotides by the guanidinium unit at the surface of aqueous micelles and bilayers. A comparison of microscopic and macroscopic interfaces. J. Am. Chem. Soc. 118, 8524-8530 (1996).

64. Ariga, K., Ito, H., Hill, J. P. \& Tsukube, H. Molecular recognition: from solution science to nano/materials technology. Chem. Soc. Rev. 41, 5800-5835 (2012). 
65. Ariga, K., Mori, T., Ishihara, S., Kawakami, K. \& Hill, J. P. Bridging the difference to the billionth-of-a-meter length scale: how to operate nanoscopic machines and nanomaterials by using macroscopic actions. Chem. Mater. 26, 519-532 (2014).

66. Ariga, K., Mori, T. \& Hill, J. P. Mechanical control of nanomaterials and nanosystems. Adv. Mater. 24, 158-176 (2012)

67. Ariga, K., Terasaka, Y., Sakai, D., Tsuji, H. \& Kikuchi, J. Piezoluminescence based on molecular recognition by dynamic cavity array of steroid cyclophanes at the air-water interface. J. Am. Chem. Soc. 122, 7835-7836 (2000).

68. Ariga, K. et al. Piezoluminescence at the air-water interface through dynamic molecular recognition driven by lateral pressure application. Langmuir 21, 976-981 (2005).

69. Michinobu, T. et al. Mechanical control of enantioselectivity of amino acid recognition by cholesterol-armed cyclen monolayer at the air-water interface. J. Am. Chem. Soc. 128, 14478-14479 (2006).

70. Mori, T. et al. Mechanical tuning of molecular recognition to discriminate the single-methyl-group difference between thymine and uracil. J. Am. Chem. Soc. 132, 12868-12870 (2010).

71. Ariga, K., Minami, K., Ebara, M. \& Nakanishi, J. What are emerging concepts and challenges in NANO?: nanoarchitectonics, hand-operating nanotechnology, and mechanobiology. Polym. J. 48, 371-389 (2016).

72. Ariga, K., Mori, T., Nakanishi, W. \& Hill, J. P. Solid surface vs. liquid surface: nanoarchitectonics, molecular machines, and DNA origami. Phys. Chem. Chem. Phys. 19, 23658-23676 (2017).

73. Shinkai, S., Ogawa, T., Nakaji, T., Kusano, Y. \& Nanabe, O. Photocontrolled extraction ability of azobenzene-bridged azacrown ether. Tetrahedron Lett. 20, 4569-4572 (1979).

74. Ishikawa, D. et al. Mechanochemical tuning of binaphthyl conformation at the air-water interface. Angew. Chem. Int. Ed. 54, 8988-8991 (2015).
75. Mori, T. et al. Mechanically induced opening-closing action of binaphthyl molecular pliers: digital phase transition vs. continuous conformational change. ChemPhysChem 18, 1470-1474 (2017).

76. Mori, T. et al. Molecular rotors confined at an ordered 2D interface. Phys. Chem. Chem. Phys. 20, 3073-3078 (2018).

77. Yonamine, Y. et al. Supramolecular 1-D polymerization of DNA origami through a dynamic process at the 2-Dimensionally confined air-water interface. Phys. Chem. Chem. Phys. 18, 12576-12581 (2016).

78. Minami, K. et al Suppression of myogenic differentiation of mammalian cells caused by fluidity of a liquid-liquid interface. ACS Appl. Mater. Interf. 9, 30553-30560 (2017).

79. Minami, K. et al. Highly ordered one-dimensional fullerene crystals for concurrent control of macroscopic cellular orientation and differentiation towards large-scale tissue engineering. Adv. Mater. 27, 4020-4026 (2015).

80. Krishnan, $\vee$. et al Vortex-aligned fullerene nanowhiskers as a scaffold for orienting cell growth. ACS Appl. Mater. Interf. 7, 15667-15673 (2015).

81. Ariga, K. Nanoarchitectonics: a navigator from materials to life. Mater. Chem. Front. 1, 208-211 (2017)

82. Wang, J., Liu, K., Xing, R. \& Yan, X. Peptide self-assembly: thermodynamics and kinetics. Chem. Soc. Rev. 45, 5589-5604 (2016).

83. Zou, Q. et al. Biological photothermal nanodots based on self-assembly of peptide-porphyrin conjugates for antitumor therapy. J. Am. Chem. Soc. 139 1921-1927 (2017).

84. Xu, Y. et al. Enhanced photophosphorylation of a chloroplast-entrapping longlived photoacid. Angew. Chem. Int. Ed. 56, 12903-12907 (2017).

85. Liu, K., Yuan, C., Zou, Q., Xie, Z. \& Yan, X. Self-assembled zinc/cystine-based chloroplast mimics capable of photoenzymatic reactions for sustainable fuel synthesis. Angew. Chem. Int. Ed. 56, 7876-7880 (2017).

86. Sun, B. et al. Self-assembly of ultralong aligned dipeptide single crystals. ACS Nano 11, 10489-10494 (2017). 